\title{
A Cooperative Clustering Protocol With Duty Cycling for Energy Harvesting Enabled Wireless Sensor Networks
}

DOI:

10.1109/TWC.2017.2762674

\section{Document Version}

Accepted author manuscript

Link to publication record in Manchester Research Explorer

\section{Citation for published version (APA):}

Bahbahani, M., \& Alsusa, E. (2017). A Cooperative Clustering Protocol With Duty Cycling for Energy Harvesting Enabled Wireless Sensor Networks. IEEE Transactions on Wireless Communications, 1.

https://doi.org/10.1109/TWC.2017.2762674

\section{Published in:}

IEEE Transactions on Wireless Communications

\section{Citing this paper}

Please note that where the full-text provided on Manchester Research Explorer is the Author Accepted Manuscript or Proof version this may differ from the final Published version. If citing, it is advised that you check and use the publisher's definitive version.

\section{General rights}

Copyright and moral rights for the publications made accessible in the Research Explorer are retained by the authors and/or other copyright owners and it is a condition of accessing publications that users recognise and abide by the legal requirements associated with these rights.

\section{Takedown policy}

If you believe that this document breaches copyright please refer to the University of Manchester's Takedown Procedures [http://man.ac.uk/04Y6Bo] or contact uml.scholarlycommunications@manchester.ac.uk providing relevant details, so we can investigate your claim.

\section{OPEN ACCESS}




\title{
A Cooperative Clustering Protocol With Duty Cycling for Energy Harvesting Enabled Wireless Sensor Networks
}

\author{
Mohammed S. Bahbahani and Emad Alsusa \\ School of Electrical and Electronic Engineering, University of Manchester, Manchester, UK
}

\begin{abstract}
This paper proposes a cooperative clustering protocol based on the low energy adaptive clustering hierarchy (LEACH) approach to enhance the longevity of energy harvesting based wireless sensor networks (EH-WSN). In the proposed protocol, to ensure that any energy consumption associated with the role of the cluster head $(\mathrm{CH})$ is shared between the nodes, the $\mathrm{CH}$ role is alternated between the nodes using duty cycling as a function of their individual energy harvesting capabilities. Furthermore, to maintain an energy neutral operation when not acting as a $\mathrm{CH}$, the nodes adopt a data transmission duty cycle and any excess energy is invested in relaying other nodes' packets. To optimize the relaying performance, a novel crosslayer cooperative TDMA scheme is also presented. The optimal number of clusters in an EH-WSN is analyzed in terms of energy consumption, latency and bandwidth utilization. Simulations, performed using GreenCastalia, demonstrate tangible performance enhancements in adopting the proposed protocol over benchmark schemes in terms of throughput and lifetime, particularly under highly constrained energy conditions.
\end{abstract}

Index Terms-Energy Harvesting, LEACH, Duty Cycle, Routing Protocol, Omnet++, Castalia, Cooperative Networks, IoT.

\section{INTRODUCTION}

Wireless Sensor Networks (WSNs) are expected to mesh a massive number of objects into what is called the Internet of Things (IoT) [1]. One of the main challenges associated with a wide deployment of WSNs is energy consumption as it is impractical to regularly replace the batteries of thousands of tiny network nodes. Therefore, extending the network lifetime is a major objective in WSN protocols [2]. Many energy conservation techniques including multi-hop, cooperative transmission and duty-cycling were proposed in the literature. Specifically, multi-hop and data aggregation, implemented through clustering, can provide energy savings at the network layer [3], whereas a periodic wake-up and sleep strategy at the MAC layer can extend the network lifetime for a certain $Q o S$ requirement as shown in [4]. Cooperative transmission at the physical layer utilizes the energy wasted in broadcast transmission by creating multiple independent paths between a source and a destination node to improve the channel capacity [5]. Despite the improvements offered by these schemes, nodes eventually die after their energies have been exhausted.

Recently, it was shown that WSN with energy harvesting (EH) capabilities, whereby nodes can harvest energy from the environment, such as solar and wind power, can sustain a perpetual lifetime [6]. Due to the random nature of such energy sources, current protocols designed for battery powered networks must be adapted to EH scenarios [7]. In response, EH clustering protocols were proposed in [8], [9], [10], [11], [12] that extend the LEACH protocol [13], in which cluster heads $(\mathrm{CH})$ aggregate and then forward data packets of their cluster members to the sink node. Unlike LEACH, which evenly distributes the $\mathrm{CH}$ role among the nodes, the aforementioned schemes elect $\mathrm{CHs}$ based on the their residual energies and forecasted harvesting rates. For instance, in [9], a $\mathrm{CH}$ decision threshold termed the energy potential $(E P)$ function is computed for each node in terms of its energy harvesting rate and current available energy as well as the potential functions of neighboring nodes. In [12], the optimal percentage of $\mathrm{CHs}$ is incorporated into a new $\mathrm{CH}$ threshold function that gets updated by the sink throughout the operation of the protocol. Specifically, a search algorithm is used by the sink to compare the current round's average throughput against that in the last round then a regulation factor is updated accordingly. The above solutions do not guarantee a perpetual operation and require the exchange of information among nodes, which creates additional overheads. The protocol in [11] proposes cluster head groups (CHG), in which nodes take turns in becoming the $\mathrm{CH}$ to minimize the overheads of the $\mathrm{CH}$ selection process. In [8], Yang et. al. analyzed an optimal multi-hop clustering architecture to achieve a perpetual operation in EH-WSNs. Particularly, energy neutrality constraints were defined and used to obtain the minimum network data transmission cycle using convex optimization. Lastly, an EH aware routing protocol based on the gradient model is proposed in [14] for WSNs. Also, a CH selection scheme based on the residual energy of nodes and their relative positions is suggested. Then, a packet forwarding mechanism is presented, that balances the energy consumption among the $\mathrm{EH}$ nodes.

Duty cycle based MAC protocols for $\mathrm{EH}$ networks were shown to maintain an Energy Neutral Operation (ENO), in which the consumed energy does not exceed the harvested energy over a given period [15]. An optimal duty cycle design is formulated in [16] as an optimization problem then solved using a low-complexity sub-optimal algorithm. An extensive analysis of different duty cycling strategies based on the battery state, harvesting rate, queue size and channel conditions was given by Niyato, et al. in [15]. Specifically, upper and lower thresholds were defined to determine the transitions between sleep and wake-up cycles. 
On the other hand, cooperative communication has been considered as a means to achieve spatial diversity gains in WSN, where installing multiple antennas on tiny nodes is infeasible. To this end, a number of researchers have investigated the problems of resource allocation and relay selection for battery powered networks [17] and EH networks [18] at the physical layer. Cooperation at the MAC layer was also shown to provide higher transmission rates and lower delays particularly in fading channels [19]. A contention based cooperative MAC protocol called CoopMAC [20] brings throughput enhancements and reduced delays in fading channels while remains compatible with the IEEE 802.11 standard. Because relaying consumes additional energy, the protocol in [21] maximizes the network lifetime by assigning scores to nodes to evaluate their availability for relaying. Another protocol in [22] incorporates two relays per source node such that the total transmission time when using both relays is less than that of the direct link. Also, a cross layer protocol that leverages cooperation at the MAC and PHY layers is presented in [23]. Specifically, a source node broadcasts its coded data bits in the first half of a time-slot, which is overheard by a potential relay node. Scheduling based cooperative MAC protocols based on time division multiple access (TDMA) were also investigated in the literature. In [24], the nodes listen in timeslots, not allocated to themselves, to the transmission of the active node and the non-acknowledgement (NACK) of the destination. Only, if a NACK is received and the active node's packet was decoded correctly, the relay node will retransmit the packet in the next allocated time slot of the following TDMA frame. A different technique is proposed in [25], where mini-slots are used to reserve cooperative resources to avoid conflicts between sources, relays and destinations. Also, cooperation enabled clustering protocols were proposed in [26], [27] and [28]. The protocol in [26] introduces cooperative cluster heads $(\mathrm{CCH})$ that are selected to maximize the spatial diversity. Non-cluster members may become $\mathrm{CCH}$ to participate in relaying the $\mathrm{CH}$ packet to the sink using a space time block code (STBC) technique. In [28], the lifetime of a battery power WSN is extended using an optimized clustering algorithm that determines the optimal locations of $\mathrm{CH}$ nodes. $\mathrm{EH}$ nodes can then be used to relay the $\mathrm{NCH}$ packets to the CHs.

In this paper ${ }^{1}$, clustering, duty cycling and cooperative transmission are combined into a novel cross-layer design for EH-WSNs. The new protocol named Energy-Harvesting and Cooperative $L E A C H$ (ECO-LEACH), modifies the LEACH technique by replacing its probabilistic $\mathrm{CH}$ selection process with a duty cycle based one to efficiently regulate the frequency at which a node undertakes the $\mathrm{CH}$ role. Besides the inherent duty cycling used by the TDMA scheduler in LEACH, another duty cycle is adopted here, by which the cluster members can skip certain allocated timeslots to maintain an ENO state. Moreover, each node follows another duty cycle to select the TDMA frames in which it is available to act as a relay. To complete the protocol, a novel cooperative

\footnotetext{
${ }^{1} \mathrm{~A}$ conference version of this paper has been accepted to appear in the International Wireless Communication and Mobile Computing Conference (IWCMC 2017). [29].
}

TDMA scheme is proposed whereby a time-slot is split into two sub-slots. All potential relays listen to the active node's transmission in the first sub-slot then the best relay transmits the received packet to the destination in the second sub-slot. The selection of the above duty cycles accounts for the node's energy harvesting rate, packet arrival rate and the optimal percentage of $\mathrm{CHs}$ in the network. Hence, a rigorous analysis of the optimal $\mathrm{CH}$ percentage (OCHP) is given, which unlike in the case of LEACH, may not necessarily minimize the network energy consumption. Instead, the optimal percentage is the one that minimizes the latency while simultaneously achieves the ENO state and bandwidth requirements. Simulations of the proposed protocol, assuming a solar energy source with random shadows, were performed using GreenCastalia [30], an extension of Castalia [31] and OMNET++ [32] simulators. The results obtained show significant improvements in throughput, latency and network lifetime compared with the conventional LEACH as well as a generic energy-aware LEACH protocol. Remarkably, these gains can be realized for both EH and battery powered networks. The contributions of this work can be listed as follows:

- Formulated the optimal $\mathrm{CH}$ percentage problem for $\mathrm{EH}$ clustering based networks that guarantees ENO, while satisfying the bandwidth and latency requirements. The problem is then solved using an iterative method for which complexity is bounded by the number of nodes in the network.

- Proposed a distributed $\mathrm{CH}$ selection scheme, using the OCHP, based on duty cycling that adapts to the energy harvesting rates. This deterministic $\mathrm{CH}$ selection in ECO-LEACH is compatible with rapidly changing energy sources such that the required $\mathrm{CH}$ percentage can be maintained over a few number of rounds. In contrast, $\mathrm{LEACH}$ requires a number of rounds equal to the number of nodes before the required $\mathrm{CH}$ percentage is maintained. Another feature of the proposed $\mathrm{CH}$ selection is the absence of harvesting rate information exchanged between the nodes as in [9]. Instead, only the average nodes' harvesting rates are required. Moreover, the proposed protocol is applicable in non-homogeneous networks, in which nodes have different capabilities and QoS requirements.

- Proposed a data transmission duty cycle to ensure ENO when the OCHP problem has no feasible solution.

- Proposed a novel TDMA-based cooperative mechanism based on sub-slots along with a relaying duty cycle design that utilizes the energy unconsumed in data transmission. The sub-slot based relaying scheme has a lower latency compared to [25], as the relayed transmission starts immediately after the direct one.

In summary, the proposed protocol first determines the OCHP (assuming nodes transmit in every allocated timeslot) since it is the most energy consuming role. If no feasible solution is found the data transmission phase of LEACH is regulated through duty cycling to maintain the ENO constraint. Any remaining energy is then invested in cooperative relaying by following another $E N O$ duty cycle. This algorithm is thus 


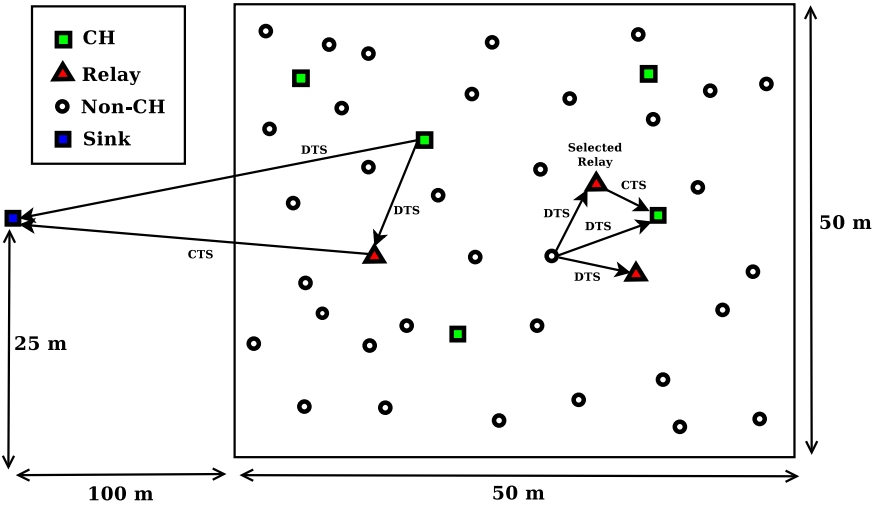

Fig. 1. Network Model

unique and to our best knowledge has not been discussed in the literature.

The remaining sections of the paper will be organized as follows. The network and energy models are defined in Section II. Section III explains the operation of the conventional LEACH protocol. The proposed ECO-LEACH is then explained in Section IV. Numerical evaluations of the proposed protocol and benchmark schemes are analyzed in Section V. Finally, concluding remarks and possible future work are stated in Section VI. Throughout the paper, the functions ceil $(x)$ and floor $(x)$ will be used to round the value $x$ to the smallest following and largest previous integer value respectively, whereas the $[x]_{0}^{1}$ limits the value of $x$ between 0 and 1.

\section{System Model And Assumptions}

\section{A. Network Model}

The network topology, as shown in Fig. 1, consists of $N=100$ stationary nodes randomly positioned in a square of dimension $M=50 \mathrm{~m}$ and a sink node placed 125 meters away from the center of the square. These positions guarantee that all nodes can reach the sink when transmitting at the maximum power level $P_{t x}^{\max }$. A log-normal shadowing radio model is assumed with its temporal variation obtained from real measurements available within the Castalia simulator. It is also assumed that the sink node transmits a short beacon at the beginning of the network operation so that each node can estimate its path loss to the sink and select the minimum required power level to reach the sink node, that is, $P_{t x}^{s i n k}$. Although node mobility is not considered in this work, the channel temporal variation created by the movement of surrounding objects is considered. The channel is assumed to be reciprocal, and its instantaneous gain can be estimated from the received signal strength indicator $(R S S I)$ value in the received packet. It is noteworthy that $R S S I$ estimation has to be longer than the channel coherence time in order to estimate the pathloss. Also, note that the pathloss estimation may need to be recalculated at defined periods of several seconds to minutes as the nodes are assumed to be stationary.

\section{B. Energy Model}

In this work, a solar energy source is assumed, which has an intensity $I_{o}$ that varies throughout the day but remains nearly constant within periods shorter than 30 minutes [16]. In addition, the average solar intensity $\bar{I}_{o}$ over a given time horizon in the future may be forecasted using the exponentially weighted moving average prediction model (EMWA) in [33]. Despite being exposed to the same intensity at any given time, each node $z$ has a different harvesting efficiency $\varepsilon_{z} \in[0,1]$. To create a more realistic scenario, it is assumed that shadows created by objects, such as clouds, may reduce the solar intensity by an opacity factor $\phi_{z}$ uniformly distributed between 0 and 1 [15]. The arrivals of shadows are modeled as a Poisson process with an inter-arrival rate $\mathcal{T}$ being constant for all nodes. This rapid fluctuation in the harvesting rate caused by the shadows is important to demonstrate the effectiveness of the proposed protocol in maintaining the required $\mathrm{CH}$ percentage over shorter periods compared to LEACH. Therefore, the overall harvesting power of a node $z$ becomes $\eta_{z}=\phi_{z} \varsigma_{z} \varepsilon_{z} I_{o}$, where $\varsigma_{z}$ is the solar cell area of node $z$. Thus, over a long period, the mean harvesting power of node $z$ becomes $\bar{\eta}_{z}=0.5 \varsigma_{z} \varepsilon_{z} \bar{I}_{o}$. Finally, the average mean harvesting power of all nodes in the network denoted by $\bar{\eta}=0.5 \overline{\zeta_{z}} \bar{\varepsilon}_{z} \bar{I}_{o}$ is assumed to be common knowledge among all nodes in the network with $\overline{\varsigma_{z}}$ and $\overline{\varepsilon_{z}}$ being the nodes average solar cell size and harvesting efficiency respectively.

\section{THE LEACH PROTOCOL}

The operation of LEACH, described in details in [13], consists of multiple rounds. Each round begins with a short setup phase followed by a long data transmission phase. During the setup phase of a round $t$, each node declares itself as a $\mathrm{CH}$ with a probability that maintains the $\mathrm{CH}$ percentage at $\pi$ after $\frac{1}{\pi}$ rounds have passed. Once a node becomes a $\mathrm{CH}$, it will never become a $\mathrm{CH}$ again until all other nodes have taken their turns. $\mathrm{CHs}$ then invite non- $\mathrm{CH}$ nodes to join their clusters by broadcasting invitation beacons. A non- $\mathrm{CH}(\mathrm{NCH})$ node joins a cluster based on the RSSI of the received beacon and selects its transmit power $P_{t x}^{C H}$ such that the received power at the $\mathrm{CH}$ is just above the sensitivity of the receiver. Upon receiving join requests, a $\mathrm{CH}$ creates then broadcasts a TDMA schedule to its cluster members.

The data transmission phase (steady state) of a round consists of multiple TDMA frames. In each time slot of a TDMA frame, a single node (active node) sends its data packet to the $\mathrm{CH}$ at a time, while other nodes switch to sleep mode. In the last timeslot of a frame, the $\mathrm{CH}$ aggregates then transmits the received packets to the sink node at power $P_{t x}^{s i n k}$. This process repeats until the round is over. To eliminate possible collisions between clusters, each cluster randomly selects a unique channel (frequency/code) from a pool of available channel resources.

Despite the performance gains over direct transmission, the uniform distribution of $\mathrm{CHs}$ does not consider the residual energy of each node. Hence, nodes that become $\mathrm{CHs}$ first will deplete their energies soon reducing network connectivity that in turn causes higher transmit power by the remaining nodes ultimately reducing the network lifetime. 


\section{Proposed Protocol Description}

The proposed ECO-LEACH protocol extends LEACH by replacing its $\mathrm{CH}$ selection process while introducing duty cycling (illustrated in Fig. 4) and cooperative transmission. Each of these features is separately discussed as follows.

\section{A. Cooperative Transmission Protocol}

In the proposed cooperative scheme, a time-slot of duration $T_{s}$ is evenly split into a direct transmission sub-slot (DTS) followed by a cooperative transmission sub-slot (CTS) as illustrated in Figures 2 and 3 above. During a DTS, the active node transmits its data packet to the $\mathrm{CH}$ and cooperating nodes. The $\mathrm{CH}$ responds with an acknowledgement (ACK) beacon if the packet was successfully received (Fig 2). Otherwise, a non - acknowledgement beacon (NACK) is sent, as shown in Fig 3. The reception of a NACK at potential relay nodes initiates a contention process, whereby a relay node replies with a relay advertisement beacon (RAB) after a delay inversely proportional to the RSSI of the received NACK. All potential relays that receive the RAB beacon, while waiting to send their RAB beacons, will back off and remain silent in the CTS sub-slot. Because some relays may be hidden from others, a relay acknowledgement beacon (RACK) is broadcasted by the destination upon receiving the first RAB so that relays will only transmit upon receiving a RACK destined to themselves [34]. The selected relay then transmits the relayed packet in the CTS sub-slot of the current time-slot. In case an ACK beacon is transmitted by the destination in the DTS, all potential relays will sleep during the CTS sub-slot. To fully utilize the allocated time-slot, the active node may transmit another data packet in the CTS as the second packet is likely to be successful without cooperation due to the correlated channels of consecutive sub-slots (Fig. 2). Clearly, the above scheme implements a decode-and-forward incremental relaying protocol [35] with the opportunistic single relay selection in [34]. It is noteworthy to mention that different cooperative schemes such as space time block code (STBC) can be implemented without affecting the above strategy. However, stringent time synchronization among relays is necessary, which is generally complex to implement.

\section{B. Cluster Head Duty Cycle Design}

Unlike the random $\mathrm{CH}$ selection in $\mathrm{LEACH}$, in this work, a node follows a $\mathrm{CH}$ duty cycle $\left(D_{C H}\right)$ that determines how often it will become a $\mathrm{CH}$ in a given time horizon $L_{h o r}$ defined as the number of rounds over which the average harvested energy can be predicted. For instance, if $D_{C H}=3$ the node becomes a $\mathrm{CH}$ only once every 3 rounds in $L_{h o r}$ as shown in Fig. 4. The $\mathrm{CH}$ duty cycle is calculated at the beginning of each $L_{h o r}$ rounds as shown in (1) at the top of the next page. In this function, $T_{r}=L_{r} T_{s}$ is the round duration, $L_{r}$ is the number of timeslots in a single round and $\alpha_{C H} \in(0,1]$ is the proportion of the harvested energy allocated to the $\mathrm{CH}$ role. Also, $E_{C H}^{r}$ is the average energy consumed by a $\mathrm{CH}$ node in a single round. When the allocated $\mathrm{CH}$ energy per round is greater than $E_{C H}^{r}, D_{C H}$ takes its minimum value of 1 .

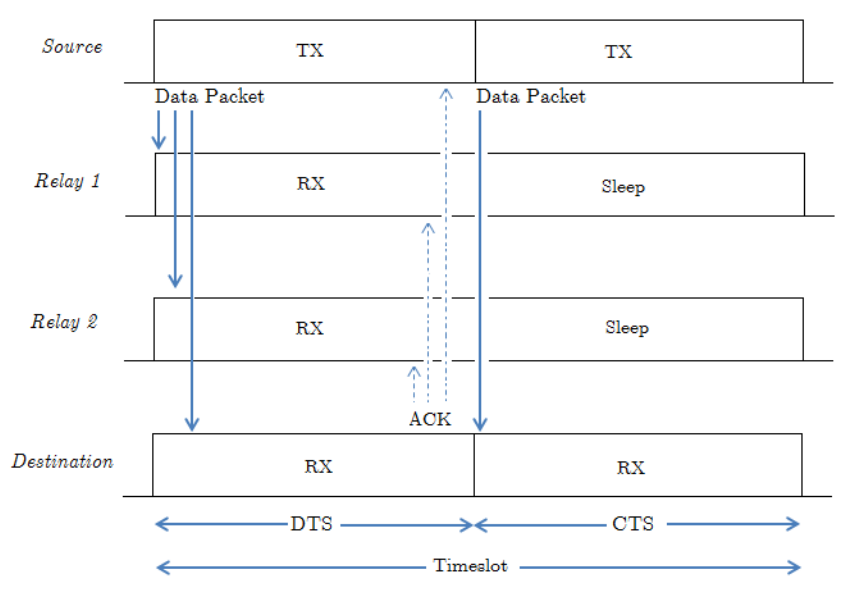

Fig. 2. Proposed Cooperative TDMA Scheme - Case 1

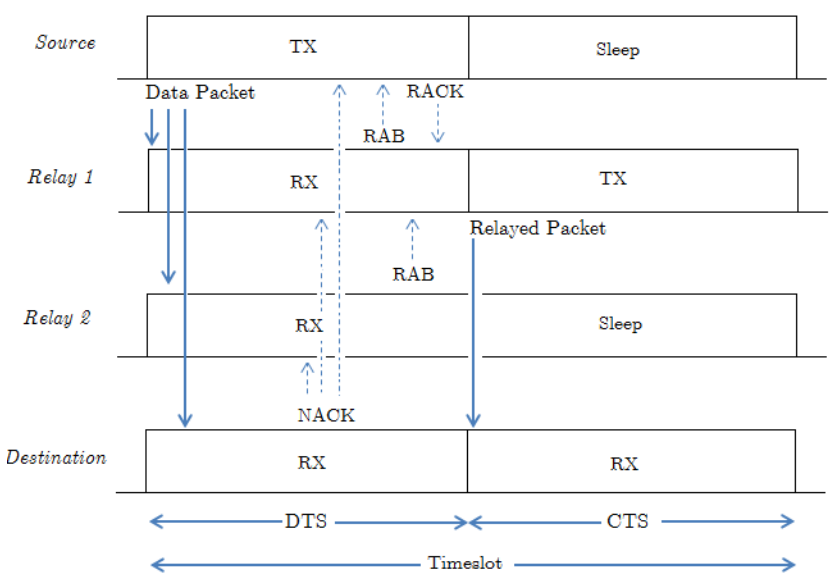

Fig. 3. Proposed Cooperative TDMA Scheme - Case 2

Otherwise, $D_{C H}$ will be the ratio of the required energy to the allocated energy rounded to the next integer value. At the start of each round, the node determines if its duty round has come using a CH-DC counter that counts up to $D_{C H}$ and then resets to 1 . To maintain the targeted percentage of $\mathrm{CHs}$, each node starts its $\mathrm{CH}-\mathrm{DC}$ counter with a random integer value between 1 and $D_{C H}$ such that 1 indicates the duty round. If a node enters the duty round with no sufficient energy, the duty round is temporarily shifted to the next round and so on. Therefore, a node's likelihood to become a $\mathrm{CH}$ in a given round is the inverse of its $D_{C H}$. Hence, to maintain the targeted percentage of CHs $\pi=\frac{k}{N}$, where $k$ denotes the number of $\mathrm{CHs}$, the factor $\alpha_{C H}$, given in (3) below, is used to limit the CH-DCs of nodes when their mean harvesting rate $\bar{\eta}$ is too high causing them to afford to turn into $\mathrm{CHs}$ more often than required. 


$$
\begin{gathered}
D_{C H}= \begin{cases}\operatorname{ceil}\left(\frac{E_{C H}^{r} L_{h o r}}{\bar{\eta}_{z} T_{r} L_{h o r} \alpha_{C H}}\right) & \frac{E_{C H}^{r} L_{h o r}}{\bar{\eta}_{z} T_{r}^{r} L_{h o r} \alpha_{C H}} \geq 1 \\
1 & \frac{E_{C H}^{r} L_{h o r}}{\bar{\eta}_{z} T_{r} L_{h o r} \alpha_{C H}}<1\end{cases} \\
E_{n e t}^{r}(k)=k\left(E_{C H}^{r}(k)+E_{N C H}^{r}(k)\right)=\frac{L_{r}}{N}\left(k^{2} P_{t x}^{s i n k} T_{s}+\left(k N-k^{2}\right)\left(P_{r x} T_{s}+E_{a g g}\right)+(N-k) T_{s} \frac{M^{2}}{2 \pi}\right)
\end{gathered}
$$

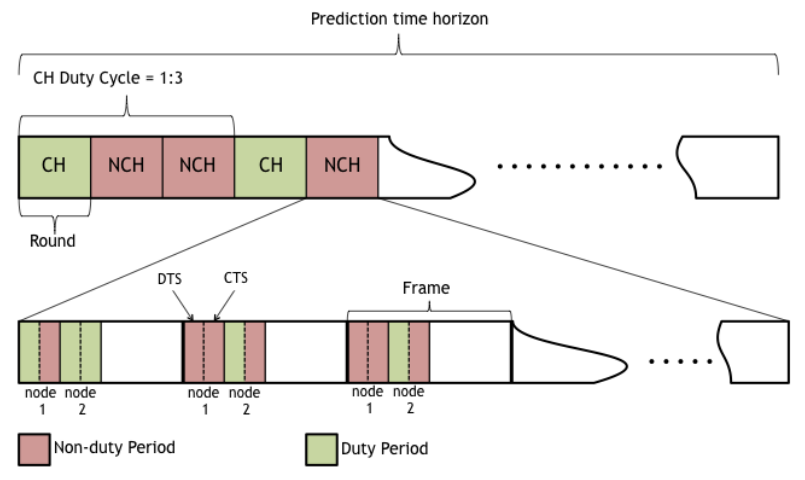

Fig. 4. Duty Cycle Structure

$$
\alpha_{C H}=\left[\frac{E_{C H}^{r} \pi}{\bar{\eta} T_{r}}\right]_{0}^{1} .
$$

\section{Optimal Cluster Head Percentage}

The number of cluster heads has different effects on the network throughput, latency, bandwidth utilization and lifetime. First, the throughput and latency affect the number of cluster heads as follows. Given the packet arrival rate $\rho$ (packets/second) and the maximum latency tolerated by the application layer $\Delta^{\max }$ (seconds), the maximum possible frame duration would be $T_{f}^{\max }=\min \left(\frac{1}{\rho}, \Delta^{\max }\right)$ leading to a minimum number of $k^{\text {min }}=\operatorname{ceil}\left(N T_{s} / T_{f}^{\max }\right)$ CHs. On the other hand, the number of available orthogonal channels defines the maximum number of clusters, $k^{\max }$, above which collisions will occur. Thus, the optimal number of CHs, $k^{o p t}$, lies in the interval $\left[k^{\min }, k^{\max }\right]$ and maintains an average network energy consumption $E_{n e t}^{r}\left(k^{o p t}\right)$ below the total energy harvested by all nodes in the network $E_{\text {har }}^{r}$ during a single round. To find $E_{n e t}^{r}(k)$, we first calculate the average energy spent by a $\mathrm{CH}$ during a given round as a function of $k$ as:

$$
E_{C H}^{r}(k)=N_{f}\left(E_{t x}^{s i n k}+\left(L_{c}-1\right) E_{r x}+E_{a g g}\right)+E_{\text {setup }},
$$

where $L_{c}=\frac{N}{k}$ is the average cluster size, $N_{f}=\frac{L_{r} k}{N}$ is the average number of TDMA frames in a given round, whereas $E_{\text {agg }}$ and $E_{\text {setup }}$ are the energies consumed in data aggregation and cluster setup respectively. Similarly, the energy needed by all $\mathrm{NCH}$ nodes per round in terms of $k$ becomes [36]:

$$
E_{N C H}^{r}(k)=N_{f}\left(L_{c}-1\right) T_{s} \bar{P}_{t x}^{C H},
$$

where the term $\bar{P}_{t x}^{C H}=\frac{M^{2}}{2 \pi k}$ approximates the average squared distance (path loss) to the $\mathrm{CH}$ assuming uniformly distributed nodes. Also, it is assumed that each $\mathrm{NCH}$ utilizes all its allocated data transmission slots. Thus, the network energy consumption is given in (2) shown at the top of the page. With more clusters (smaller average cluster size) the node-tonode distance is reduced causing less energy spent by the $\mathrm{NCH}$ nodes in conveying their data packets to their CHs. However, data aggregation is reduced and more energy consuming $\mathrm{CHs}$ are introduced. Therefore, the OCHP is unique to the network topology and parameters, which can be formulated as:

$$
k^{o p t}=\max k
$$

s.t.

$$
\begin{gathered}
E_{n e t}^{r}(k) \leq E_{h a r}^{r}=L_{r} T_{s} \sum_{z=1}^{N} \eta_{z} \\
k \leq k^{\max }, k \geq k^{\text {min }} \\
k \in\{1, \ldots, N\}
\end{gathered}
$$

The optimization problem above gives the highest $k$ (maximum spectral efficiency) that maintains the ENO (constraint (7)) for any value of $k$ in the range defined by constraints (8) and $(9)^{2}$. Unlike the OCHP analysis in [36], the proposed optimal solution may not necessarily minimize the network energy consumption, as some values of $k \neq k^{\text {opt }}$ may result in a lower energy consumption. However, these values may degrade the system performance, since by having more clusters than orthogonal channels, backoffs induced by CSMA at the MAC layer may result in longer delays and more collisions. Conversely, choosing a value of $k$ below $k^{\text {min }}$ leads to more dropped packets due to buffer overflow and timeouts.

The solution to the non-linear integer programming problem formulated above can be centrally obtained by evaluating $E_{n e t}^{r}(k)$ starting from $k^{\max }$ until a value that satisfies the constraint in (7) is found. Thus, the solution has a linear complexity in the number of nodes. However, a feasible solution may not exist if $E_{n e t}^{r}(k)>E_{h a r}^{r} \forall k \in\left\{k^{m i n}, k^{m i n}+\right.$ $\left.1, \ldots, k^{\max }-1, k^{\max }\right\}$, in which case reducing the data transmission duty cycle is necessary as will be discussed next.

\footnotetext{
${ }^{2}$ It is assumed that the network parameters are set such that $k^{\text {min }} \leq k^{\text {max }}$ and $k^{\min }, k^{\max } \in\{1, \ldots, N\}$.
} 
Due to the absence of a central station in many WSN scenarios, the OCHP can be obtained in a distributed fashion, where each node independently determines $k^{o p t}$ assuming the knowledge of the network parameters and the mean average energy harvesting $\bar{\eta}$ instead of $\sum_{z=1}^{N} \eta_{z}$ in (7). Each node then substitutes $k^{o p t} / N$ in (3) to find its CH-DC. Henceforth, the OCHP will refer to the distributed OCHP. As the harvesting rate changes with time, the OCHP is dynamically updated at the beginning of each $L_{h o r}$ period as will be demonstrated in Section V.

\section{Data Transmission Duty Cycle Design}

A periodic wake-up/sleep strategy is inherently implemented in TDMA, as nodes sleep in non-allocated timeslots. In $\mathrm{LEACH}$, this will cause each cluster member to undergo an average duty cycle of $1: L_{c}$. However, if the predicted harvested energy is still insufficient to maintain an $E N O$, the duty cycle should be further reduced by skipping the allocated slot in certain TDMA frames. In addition, the duty cycle should also adapt to the packet arrival rate $\rho$ since switching to transmit mode with no data packet to send results in an unnecessary energy waste. Hence, a data transmission duty cycle $D_{D T}$, based on the harvesting power and the packet arrival rate, is proposed that defines the number of TDMA frames to skip after each data transmission. For example, in Fig. 4, node 1 utilizes its allocated slot only once every 3 TDMA frames, hence its $D_{D T}$ is 3 , whereas node 2 with $D_{D T}=1$ uses its timeslot in every frame. A node $z$ computes its $D_{D T}$ at the beginning of every round by first finding the expected remaining harvested energy per $\mathrm{NCH}$ round given as:

$$
E_{N C H}^{r e m}=\left(\bar{\eta}_{z} T_{s} L_{r} L_{h o r}-E_{C H}^{r}\left(L_{h o r}-L_{N C H}\right)\right) / L_{N C H}
$$

where $L_{N C H}=L_{h o r}-\frac{L_{h o r}}{D_{C H}}$ is the number of NCH rounds in $L_{h o r}$. The node then computes a duty cycle with respect to the harvesting rate and another for the data arrival rate as:

$$
\begin{gathered}
D_{D T}^{e n e}=\left\{\begin{array}{ll}
\operatorname{ceil}\left(\frac{E_{t x}^{C H} N_{f}}{E_{N C H}^{r e m}}\right) & 1 \leq\left(\frac{E_{t x}^{C H} N_{f}}{E_{N C H}^{r e m}}\right) \leq N_{f} \\
1 & \left(\frac{E_{t x}^{C H} N_{f}}{E_{N C H}^{r e m}}\right)<1
\end{array},\right. \\
D_{D T}^{\text {data }}=\left\{\begin{array}{ll}
\operatorname{floor}\left(\frac{N_{f}}{\rho T_{r}}\right) & 1 \leq\left(\frac{N_{f}}{\rho T_{r}}\right) \leq N_{f} \\
1 & \left(\frac{N_{f}}{\rho T_{r}}\right)<1
\end{array},\right.
\end{gathered}
$$

where $E_{t x}^{C H}=P_{t x}^{C H} T_{s}$ is the energy consumed in transmitting a packet to the $\mathrm{CH}$. Hence, $D_{D T}$ is found as:

$D_{D T}= \begin{cases}0 & \max \left(D_{D T}^{e n e}, D_{D T}^{\text {data }}\right)>N_{f} \\ \max \left(D_{D T}^{\text {ene }}, D_{D T}^{\text {data }}\right) & 1 \leq \max \left(D_{D T}^{\text {ene }}, D_{D T}^{\text {data }}\right) \leq N_{f}\end{cases}$

Similar to the CH-DC design, when the average energy available in an $\mathrm{NCH}$ round is greater than that consumed when the node transmits in every frame in the round and that the number of generated packets is greater than $N_{f}$, then $D_{D T}$ is set to 1 . Conversely, if the energy available for data transmission is less than that needed for a single data transmission or the number of generated packets is less than 1, the node will not join any $\mathrm{CH}$ and will remain silent in the whole round, that is, $D_{D T}=0$. For any intermediate values, the node will be active once every $D_{D T}$ frames. Again, a DC counter is employed to a keep track of the duty cycle as in CH-DC.

\section{E. Relaying Duty Cycle Design}

In certain TDMA frames, a node may act as a potential relay according to the cooperative strategy explained in Section IV-A. Particularly, the node may relay packets of other cluster members to the $\mathrm{CH}$ during their allocated time-slots at power $P_{t x}^{C H}$ and may also relay the aggregated packet of the $\mathrm{CH}$ to the sink at power $P_{t x}^{s i n k}$. Hence, we define the relaying duty cycle $D_{R L}$ as the number of frames in which the node becomes a potential relay only once. According to the example in Fig. 4, node 1 never acts as a relay and hence its relaying DC is zero, whereas the $D_{R L}$ of node 2 is 3 . The relaying duty cycle is computed at the beginning of each round, after calculating $D_{D T}$, by first finding the remaining energy from $E_{N C H}^{r e m}$, after subtracting the energy reserved for data transmission, as:

$$
E_{N C H-R}^{r e m}=E_{N C H}^{r e m}-\frac{N_{f}}{D_{D T}} E_{t x}^{C H}
$$

Thus, the relay transmission duty cycle can be given as:

$$
D_{R T}= \begin{cases}0 & \frac{E_{R L}^{f} N_{f}}{E_{N C H}^{r e m}-R}>N_{f} \\ \operatorname{ceil}\left(\frac{E_{R L}^{f} N_{f}}{E_{N C H-R}^{r e m}}\right) & 1 \leq \frac{E_{R L}^{f} N_{f}}{E_{N C H-R}^{r e m}} \leq N_{f} \\ 1 & \frac{E_{R L}^{f} N_{f}}{E_{N C H-R}^{r e m}}<1\end{cases}
$$

where $E_{R L}^{f}=P_{r x}\left(L_{c}-1\right) \frac{T_{s}}{2}+P_{t x}^{C H}\left(L_{c}-2\right) \frac{T_{s}}{2}+P_{t x}^{S i n k} \frac{T_{s}}{2}$ is the energy needed by a node to act as a relay during a single frame. Similar to the above DCs, a relaying DC counter is employed.

\section{Simulations}

The proposed protocol was simulated using Castalia, an extension of OMNET++. The default log-normal shadowing radio model of Castalia was used with a path loss exponent of 2.0. In addition, the temporal channel model of Castalia, based on real channel measurements, was adopted to demonstrate the spatial diversity gains of cooperative transmission. The transceiver $C C 1000$ was assumed at the physical layer, which allows for transmit power levels ranging from -20 $\mathrm{dB}$ to $10 \mathrm{~dB}$ consuming 15.9 to $80.1 \mathrm{~mW}$ respectively. The sensitivity of the chip is $-95 \mathrm{dBm}$, while the receive power consumption is $22.2 \mathrm{dBm}$. In sleep mode, the chip consumes only $0.6 \mu \mathrm{W}$ and transitions between states take up $0.2 \mathrm{~ms}$ consuming power up to $0.5 \mu \mathrm{W}$. At the MAC layer, the conventional CSMA protocol with an exponential back off was adopted, which was necessary to avoid collisions during cluster setup [13]. The parameters of ECO-LEACH and the topology settings, are specified in Table I, were used in all simulations unless stated otherwise. The energy harvesting model 
was implemented using GreenCastalia [30], an extension to Castalia that simulates energy sources, storage, harvesters and managers. Each node has a single ideal rechargeable battery with a capacity of $3 \mathrm{mAh}^{3}$ initially charged with $5 \%$ of its full capacity, whereas the sink node was assumed to be nonenergy constrained. A single solar energy source was used with an intensity defined per simulated scenario. In addition, the mean arrival rate of shadows $\mathcal{T}$ was set to 1 second. Further, the solar cell efficiency of each node was randomly selected with a mean of 0.22 . The proposed algorithms including the proposed protocol and other benchmark schemes are briefly described as follows:

- Conventional LEACH (C-LEACH):

This simulated version of LEACH follows the protocol described in Section III with a few modifications. First, in each time-slot, only a single data packet may be transmitted occupying the whole duration of the time-slot [15], as this approach is used to simplify the simulation of the proposed cooperative protocol below. Also, a node can only declare itself a $\mathrm{CH}$ if an amount of $E_{C H}^{r}$ is available in its battery. In addition, the node can only transmit a packet if a minimum of $P_{t x}^{C H} T_{s}$ of energy is available.

- Energy-Aware LEACH (EA-LEACH):

Instead of comparing the proposed solution to specific $\mathrm{EH}$ protocols in the literature, a generic energy-aware LEACH termed EA-LEACH was simulated. This is due to the protocols in the literature having different energy models and assumptions. Moreover, these reference protocols were evaluated in Matlab, which is less accurate in simulating upper layer protocols. In this generic protocol, the $\mathrm{CH}$ selection incorporates the energy harvesting status of all nodes in the network such that nodes with more relative harvesting rates becomes $\mathrm{CHs}$ more often with no restriction on the number of times a node can become a $\mathrm{CH}$. Hence, a node $z$ becomes a $\mathrm{CH}$ based on the following probability function:

$$
\mathcal{P}_{z}(t)=\frac{N \pi\left(E_{z}^{b a t}+E_{z}^{h a r}\right)}{\sum_{i=1}^{N} E_{i}^{\text {bat }}+E_{i}^{\text {har }}},
$$

where $E_{i}^{b a t}$ and $E_{i}^{h a r}$ are the residual energy and harvesting rate of node $i$.

- Proposed Protocol Without Cooperation (ENCOLEACH):

The proposed protocol described in Section IV is simulated without the cooperation feature in ENCO-LEACH. Hence, this protocol only benefits from the proposed $\mathrm{CH}$ selection and data transmission duty cycles as well as the OCHP derived in Section IV-C.

- Proposed Protocol With Cooperation (ECO-LEACH):

Here, the full proposed protocol including cooperative diversity is simulated. Since data packets are sent with half the number of bits during a sub-slot, twice the number of packets are generated with respect to the other protocol to ensure a fair comparison.

\footnotetext{
${ }^{3}$ The small capacity used is due to the relatively short simulation time (5000 sec) compared with the real deployment.
}

Table I

SimULATION PARAMETERS

\begin{tabular}{|c|c|c|}
\hline$N$ & 100 & Number of Nodes \\
\hline$T_{s}$ & $100 \mathrm{~ms}$ & Time slot Duration \\
\hline$L_{r}$ & 500 timeslots & Round Length \\
\hline$L_{h o r}$ & 10 rounds & Prediction Time Horizon \\
\hline$E_{a g g}$ & $5 \mathrm{~nJ} / \mathrm{bit}$ & Aggregation Energy \\
\hline$\varsigma_{z}$ & $1.54 \mathrm{~cm}^{2}$ & Solar Cell Size \\
\hline$\rho$ & $0.5 \mathrm{packet} / \mathrm{s}$ & Data Packet rate \\
\hline$T_{\text {sim }}$ & $5000 \mathrm{~s}$ & Simulation time \\
\hline$\Delta_{\max }$ & $5 \mathrm{~s}$ & Max. Delay \\
\hline$\theta$ & 20 & Number of Orthogonal Channels \\
\hline & $2000 \mathrm{bit}$ & Data Payload \\
\hline
\end{tabular}
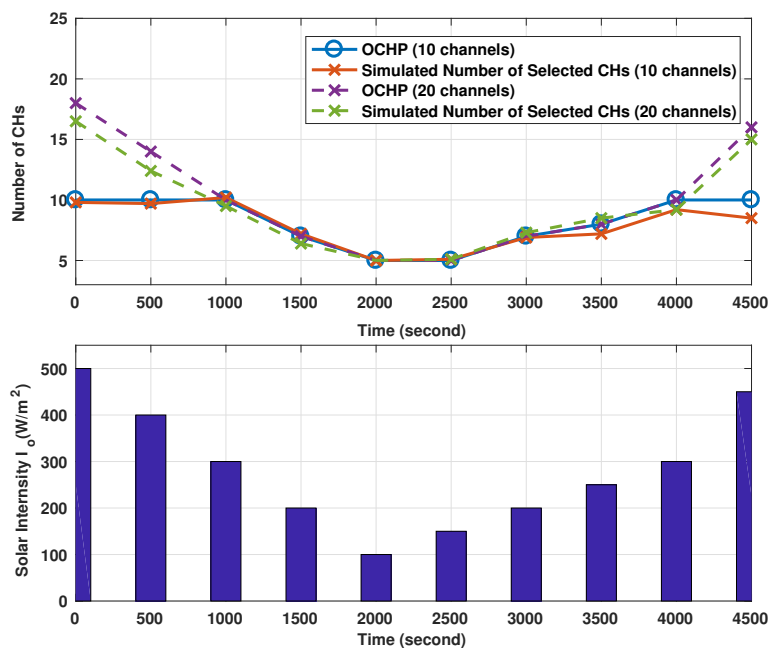

Fig. 5. Optimal CH Percentage for Different Solar Intensities with $\rho=0.5$

Different scenarios averaged over 1000 iterations were created to evaluate the performance of the above protocols based on the throughput, latency and lifetime as shown below. In this context, the average network throughput is defined as the number of packets successfully delivered to the sink, within the max delay period, divided by the total number of packets generated by all nodes over the simulation period $\left((N-1) T_{\text {sim }}\right)$. For example, if the packet arrival rate is 1 packet/second, a throughput of $40 \%$ means that 2000 packets were ultimately delivered to the sink by each node on average. Since the energy consumption is always maintained below the harvested energy in an ENO, maximizing the throughput also maximizes the energy efficiency. As for the lifetime, it is defined as the time until the first node in the network depletes its battery.

The first objective was to verify the OCHP solution proposed in Section IV-C. As depicted in Fig. 5, the OCHP follows the harvesting power (and solar intensity) within the feasible range defined by the maximum number of orthogonal channels (10 and 20 channels) and the packet arrival rate of 0.5 packet $/ \mathrm{sec}^{4}$. The simulated number of $\mathrm{CHs}$ selected in the proposed protocol is shown to closely follow the OCHP for all intensities. The small error seen is due to some nodes being unable to become $\mathrm{CHs}$, after being selected, due to insufficient

\footnotetext{
${ }^{4} \mathrm{~A}$ low packet arrival rate is typical in many surveillance and environmental monitoring WSN applications [37].
} 


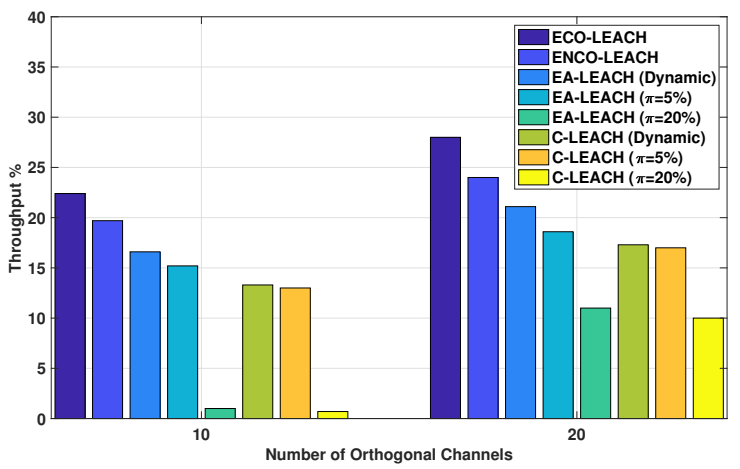

Fig. 6. Dynamic vs. Static CH Percentage with $\rho=0.5$

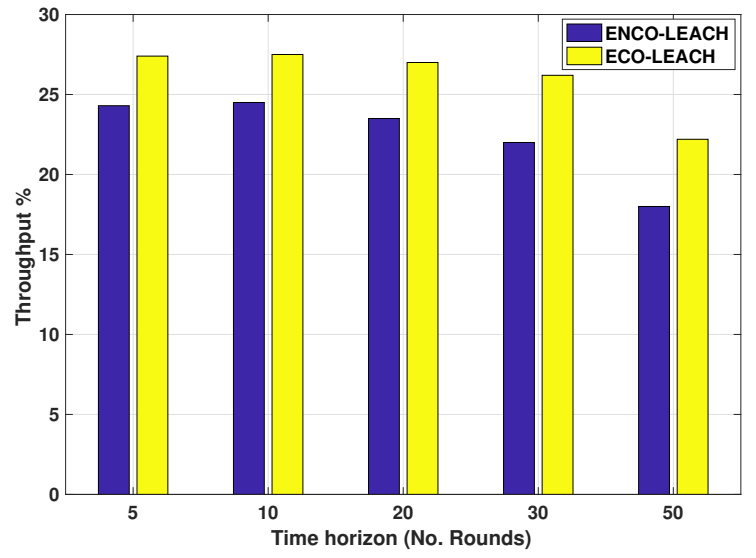

Fig. 7. Average Network Throughput vs. Time Horizon

residual energies. Specifically, in the period 0 to 1000 s, the optimal $\mathrm{CH}$ number drops from 17 to $10 \mathrm{CHs}$ as the harvested energy is not enough to support $20 \mathrm{CHs}$ even when 20 channels are available. However, with 10 available channels, the number of CHs saturates at 10 for the same period since $k_{\max }=10$. The $\mathrm{CH}$ number falls as the energy harvested is further reduced between 1000s and 2500s. Since the number of CHs supported is below 10 , the available channels, being 10 or 20 , cannot be utilized and thus the curves overlap. The reverse is then observed when the harvested energy increases from 2500s onward.

The throughput of the proposed protocol with the dynamically computed OCHP compared against the benchmark protocols, equipped with either static or dynamic $\mathrm{CH}$ percentage, is depicted in Fig. 6 for different number of orthogonal channels using the same intensity distribution in Fig 5. Both C-LEACH and EA-LEACH are seen to benefit from the dynamic OCHP compared to static $\mathrm{CH}$ percentage fixed at extreme values of $5 \%$ and $20 \%$. Also, a sharp fall in throughput is seen for the static $20 \%$ case as the number of selected $\mathrm{CH}$ is far more than the number of available orthogonal channels. Notably, the proposed protocols outperform the benchmark ones even when they adopt the proposed dynamic OCHP mechanism.

In Fig. 7, the throughput of the proposed protocol is analyzed for several time horizon values, assuming the same intensity distribution above. By extending the time horizon beyond 10 rounds, the performance starts to deteriorate, as the actual energy harvested during each round deviates from the predicted value used to compute the $\mathrm{CH}-\mathrm{DC}$ at the beginning of each $L_{h o r}$ period. Consequently, the harvested energy will not be optimally invested in maintaining the ENO. Note that the solar intensity in Fig 5 remains constant for 10 rounds. Conversely, making the time horizon unnecessarily short will increase the $\mathrm{CH}$ setup overheads, which in turn, reduce the network throughput.

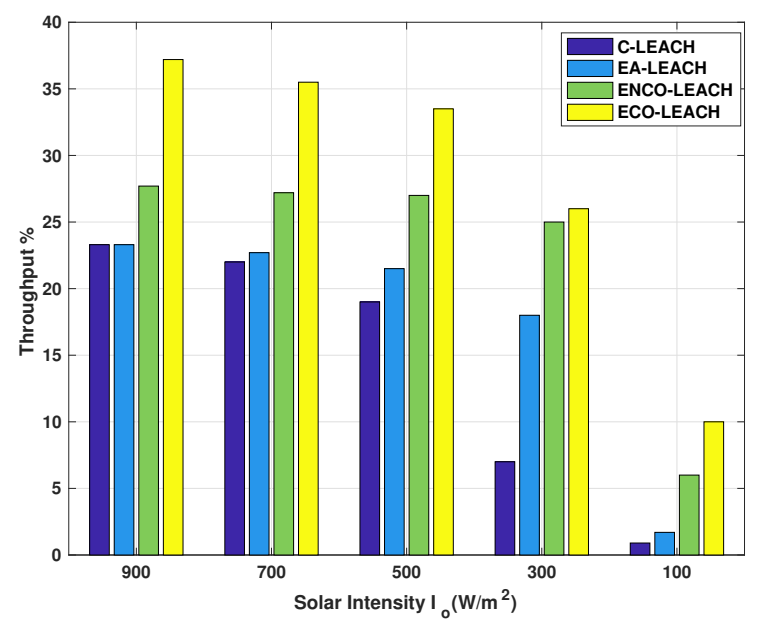

Fig. 8. Average Network Throughput vs. Solar Intensity $(\theta=10, \rho=0.5)$

Fig. 8 compares the network throughput of the proposed protocol with and without cooperation against the benchmark protocols for different solar intensities. At high intensities, it is seen that EA-LEACH has a negligible advantage over C-LEACH as almost all nodes are capable of becoming $\mathrm{CHs}$, whereas the proposed ENCO-LEACH outperforms the aforementioned protocols by around 19\% due to its DT-DC. A further gain of $35 \%$ is obtained when utilizing cooperative relaying . At $300 \mathrm{~W} / \mathrm{m}^{2}$, the performance of ECO-LEACH is seen to fall to near that of ENCO-LEACH as less energy is left for relaying after allocating energy to the $\mathrm{CH}$ and data transmission roles. In turn, fewer relays will be available in each time slot reducing the diversity gain and hence the channel capacity. At lower intensities, however, the small portion of energy allocated to relaying has a more significant effect on the throughput as clusters tend to be fewer and larger in size. When this occurs, the average $\mathrm{NCH}$ to $\mathrm{CH}$ distance increases making the direct transmission less reliable. It is also evident that the gains achieved by the proposed protocol over C-LEACH and EA-LEACH are more significant at lower solar intensities. Specifically, a gain of nearly $500 \%$ is achieved by ECO-LEACH with respect to EA-LEACH at $100 \mathrm{~W} / \mathrm{m}^{2}$ compared with only $60 \%$ at $900 \mathrm{~W} / \mathrm{m}^{2}$.

The effect of varying the data packet arrival rate on the performance of the different simulated protocols is shown in Fig. 9 for a fixed solar intensity of $500 \mathrm{~W} / \mathrm{m}^{2}$. In general, all the protocols perform worse with higher packet arrival rates due to the relatively low harvesting rate which limits the number of packets that can be transmitted. However, 


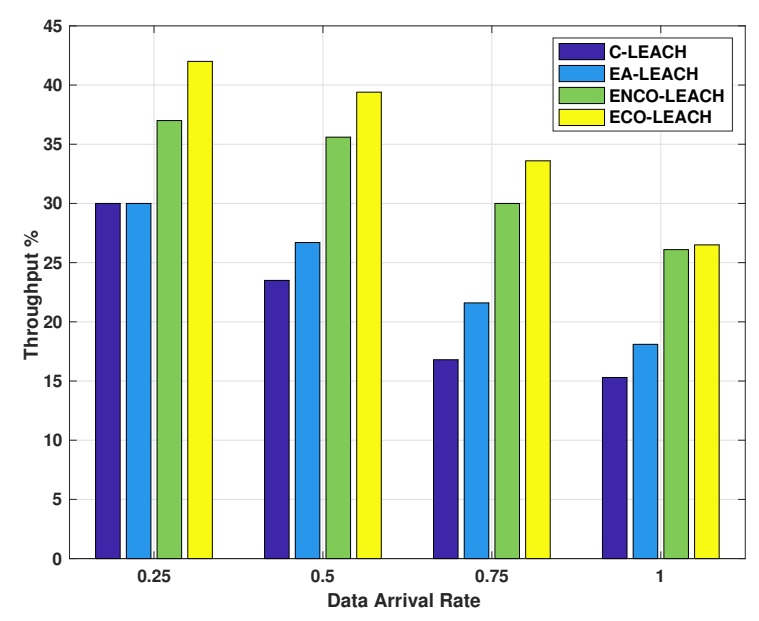

Fig. 9. Average Network Throughput vs. Packet Arrival Rate $\left(\theta=20, I_{o}=\right.$ $500 \mathrm{~W} / \mathrm{m}^{2}$ )

the throughput of the proposed protocol drops by only $26 \%$ when the arrival rate is doubled compared to a $32 \%$ loss by EA-LEACH. It is also seen that the gain from cooperation diminishes at higher packet arrival rates due to the lower relaying duty cycle resulting from a relatively higher data transmission DC.

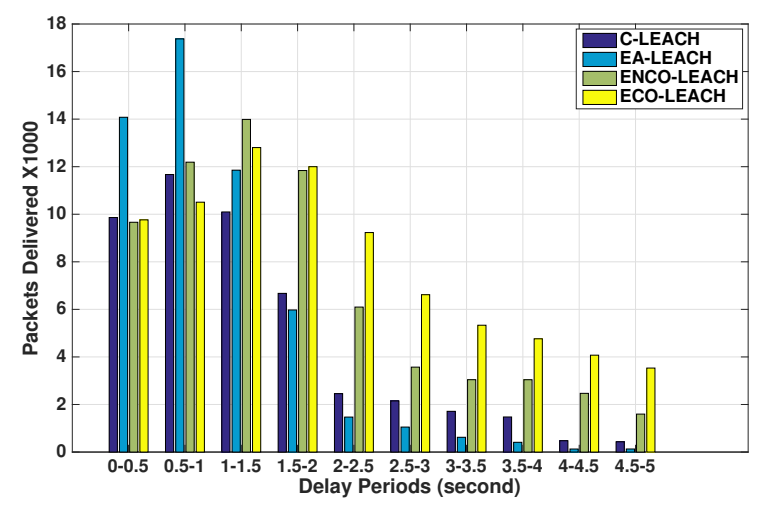

Fig. 10. Number of delivered packets vs. Delay periods $(\theta=20, \rho=0.5$, $I_{o}=500 \mathrm{~W} / \mathrm{m}^{2}$ )

The latency in delivering packets to the sink node is evaluated for the different protocols in Fig 10, assuming a solar intensity of $500 \mathrm{~W} / \mathrm{m}^{2}$. It is shown that the proposed protocol introduces longer delays than the benchmark schemes. This is explained by the reduced data transmission DC in order to adapt to the harvesting rate and maintain the ENO. It is also seen that cooperation introduces a further delay as failed packets are not transmitted until the following sub-slots. Despite that, more packets are delivered using the proposed protocol within the maximum delay period of 5 seconds, leading to a higher throughput as the energy is evenly spread throughout the duration of the simulation.

The effect of increasing the number of nodes and hence the network density on the performance of ECO-LEACH is demonstrated in Fig. 11. Generally, increasing the nodes

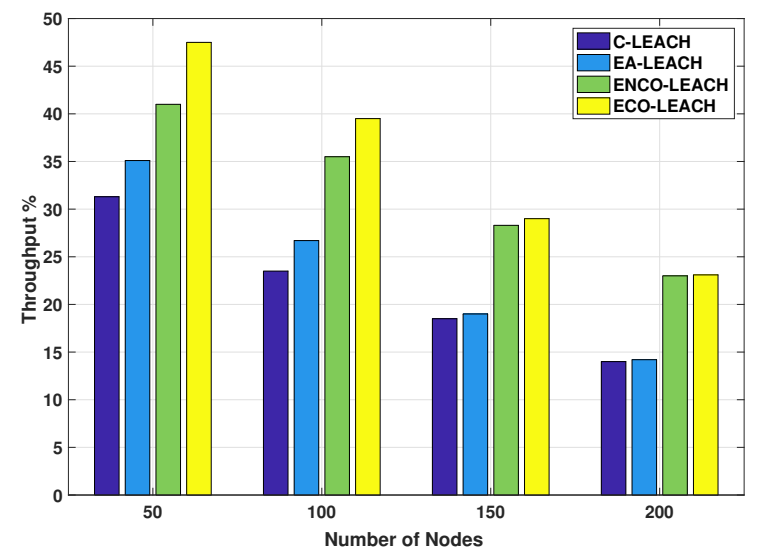

Fig. 11. Average Network Throughput vs. Number of Nodes $(\theta=20, \rho=$ $0.5, I_{o}=500 \mathrm{~W} / \mathrm{m}^{2}$ )

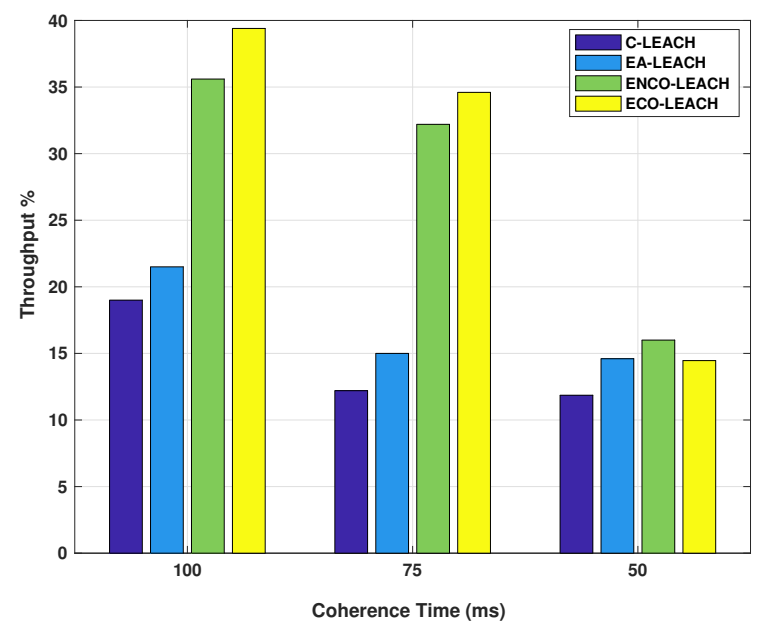

Fig. 12. Average Network Throughput vs. Channel Coherence Time $(\theta=$ $20, \rho=0.5, I_{o}=500 \mathrm{~W} / \mathrm{m}^{2}$ )

degrades the average throughput in all protocols due to larger clusters formed (as $k_{\max }$ is limited by the available channels) and thus longer delays. Also, the highly dense collision domain leads to more backoffs and collisions. Meanwhile, the gain obtained from the proposed protocol is more evident at high $N$ as the reduced DT duty cycle lowers the effect of collisions and interference. However, the gain from cooperation diminishes as $N$ increases since the reduced node-to-node distance decreases the spatial diversity gain.

To evaluate the impact of the fading channel on the performance of the proposed protocol, throughput simulations were run with different channel coherence times as shown in Fig. 12. The shorter the coherence time of the channel the worse is the network performance in all protocols, as the $R S S I$ based $\mathrm{CH}$ selections will turn invalid after the channel changes during a single round of the protocol. It is also seen that the cooperative protocol's performance drops with shorter coherence times until it becomes worse than ENCO-LEACH at $T_{c}=50 \mathrm{~ms}$. Again, this is explained by the fact that the best relay selected in the DTS sub-slot may not be the best 


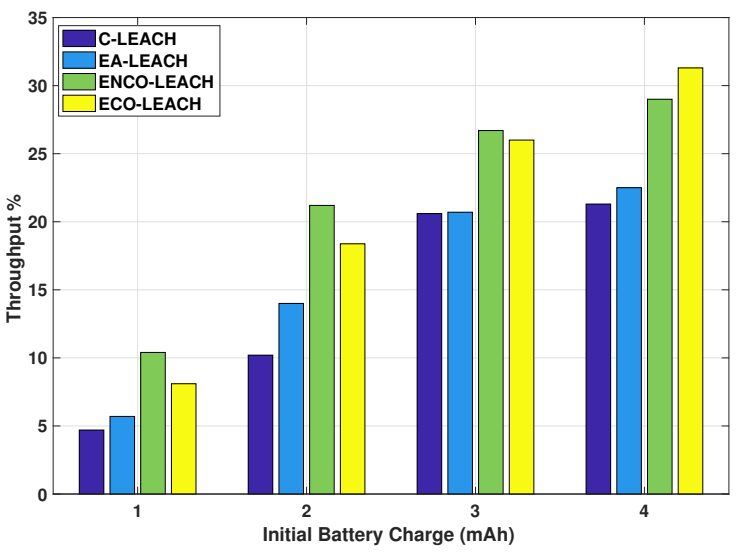

Fig. 13. Average Network Throughput vs. Initial Battery Charge

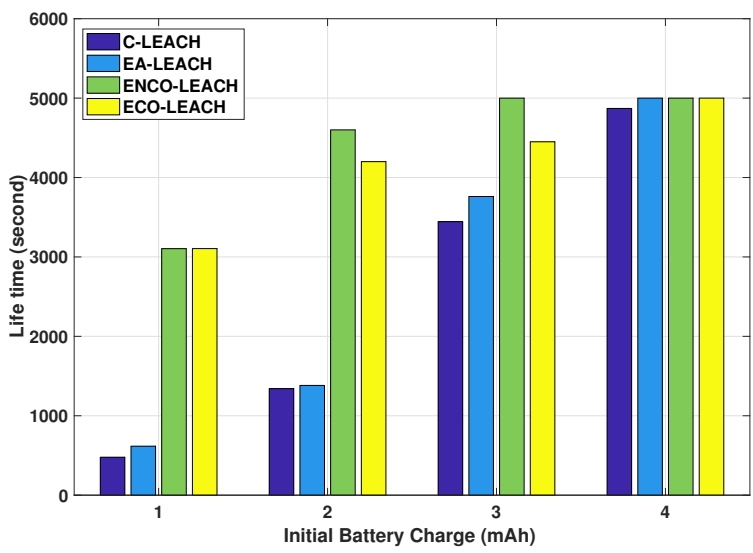

Fig. 14. Network Lifetime vs. Initial Battery Charge

relay in the following $R T S$.

Finally, figures 13 and 14 evaluate the performance of the proposed protocol with battery powered nodes in terms of throughput and lifetime. In this scenario, $L_{h o r}$ is set to equal the simulation duration and the expected harvested energy $\bar{\eta}_{\mathrm{z}} T_{r} L_{h o r}$ in all duty cycle calculations is replaced by the initial battery charge. It is seen that the proposed protocols outperform the benchmark schemes for all values of initial energy due to the novel duty cycling and cooperative schemes. Specifically, at a low initial charge, the throughput is nearly doubled by using the proposed scheme, whereas the lifetime is extended by almost 5 folds. As the initial charge is increased, the performance of ENCO-LEACH and the benchmark protocols become closer as they all reach the maximum lifetime of $T_{\text {sim }}$ at 4 (mAh) of initial charge. However, the proposed scheme is still superior in terms of throughput due to the DT-DC. As for ECO-LEACH, it is seen that no gain from cooperation is obtained for low values of initial residual energy. This is because all the available energy is used for the node's own data transmissions as it is given priority over relaying others packets. The small degradation in the performance of ECO-LEACH at low initial charge is due to the doubling of the packet generation rate as described above.

\section{CONCLUSION}

A novel clustering protocol that incorporates duty cycling and cooperative transmission is proposed for energy harvesting WSNs. The duty cycle for the $\mathrm{CH}$ assignment that guarantees a targeted $\mathrm{CH}$ percentage was derived and its optimal value was investigated. An efficient DC that ensures a perpetual network operation was developed. Besides, a cross-layer cooperative transmission strategy was designed to enable nodes to relay undelivered packets from cluster members to $\mathrm{CHs}$ and also from $\mathrm{CHs}$ to the sink node. The results obtained using event driven simulations have demonstrated an enhanced network performance in terms of throughput and lifetime with respect to the conventional LEACH as well as a generic energy-aware LEACH in EH and conventional battery powered WSNs. As a future development, this protocol can be extended to include the node's position and mobility in the duty cycle designs. In addition, hierarchical and multi-hop clustering can be integrated to support large network deployments such as the $I o T$.

\section{REFERENCES}

[1] Q. Chi, H. Yan, C. Zhang, Z. Pang, and L. D. Xu, "A reconfigurable smart sensor interface for industrial WSN in IoT environment," IEEE Trans. Ind. Inf., vol. 10, no. 2, pp. 1417-1425, May 2014.

[2] N. A. Pantazis, S. A. Nikolidakis, and D. D. Vergados, "Energy-efficient routing protocols in wireless sensor networks: A survey," Commun. Surveys Tuts., vol. 15, no. 2, pp. 551-591, Second 2013.

[3] C. Wei, J. Yang, Y. Gao, and Z. Zhang, "Cluster-based routing protocols in wireless sensor networks: A survey," in International Conf. Comput. Sci. and Netw. Technol. (ICCSNT), 2011, vol. 3, Dec 2011, pp. 16591663.

[4] O. Yang and W. Heinzelman, "Modeling and performance analysis for duty-cycled MAC protocols with applications to S-MAC and X-MAC," IEEE Trans. Mobile Comput., vol. 11, no. 6, pp. 905-921, June 2012.

[5] M. Bahbahani, M. Baidas, and E. Alsusa, "A distributed political coalition formation framework for multi-relay selection in cooperative wireless networks," IEEE Trans. Wireless Commun., vol. 14, no. 12, pp. 6869-6882, Dec. 2015.

[6] J. Zheng, Y. Cai, X. Shen, Z. Zheng, and W. Yang, "Green energy optimization in energy harvesting wireless sensor networks," IEEE Commun. Mag., vol. 53, no. 11, pp. 150-157, November 2015.

[7] S. Sudevalayam and P. Kulkarni, "Energy harvesting sensor nodes: Survey and implications," Commun. Surveys Tuts., vol. 13, no. 3, pp. 443-461, 2011.

[8] L. Yang, Y. Lu, Y. Zhong, X. Wu, and S. X. Yang, "A multi-hop energy neutral clustering algorithm for maximizing network information gathering in energy harvesting wireless sensor networks," Sensors, vol. 16, no. 1, p. 26, 2016. [Online]. Available: http://www.mdpi.com/1424-8220/16/1/26

[9] M. Xiao, X. Zhang, and Y. Dong, "An effective routing protocol for energy harvesting wireless sensor networks," IEEE Wireless Commun. Netw. Conf. (WCNC), pp. 2080-2084, April 2013.

[10] S. M. Bozorgi, M. G. Amiri, A. S. Rostami, and F. Mohanna, "A novel dynamic multi-hop clustering protocol based on renewable energy for energy harvesting wireless sensor networks," 2015 2nd International Conf. on Knowledge-Based Engineering and Innovation (KBEI), pp. 619-624, Nov 2015.

[11] S. Peng and C. P. Low, "Energy neutral clustering for energy harvesting wireless sensors networks," IEEE International Conf. Netw. (ICON), pp. 1-6, Dec 2013

[12] J. Meng, X. Zhang, Y. Dong, and X. Lin, "Adaptive energy-harvesting aware clustering routing protocol for wireless sensor networks," International Conf. on Commun. and Netw. in China (CHINACOM), pp. 742747, Aug 2012. 
[13] W. R. Heinzelman, A. Chandrakasan, and H. Balakrishnan, "Energyefficient communication protocol for wireless microsensor networks," in Proceedings of the 33rd Annual Hawaii International Conf. on Syst. Sci., Jan 2000, pp. 10 pp. vol.2-.

[14] D. Wu, J. He, H. Wang, C. Wang, and R. Wang, "A hierarchical packet forwarding mechanism for energy harvesting wireless sensor networks," IEEE Commun. Mag., vol. 53, no. 8, pp. 92-98, Aug 2015.

[15] D. Niyato, E. Hossain, and A. Fallahi, "Sleep and wakeup strategies in solar-powered wireless sensor/mesh networks: Performance analysis and optimization," IEEE Trans. Mobile Comput., vol. 6, no. 2, pp. 221-236, Feb 2007.

[16] J. Hsu, S. Zahedi, A. Kansal, M. Srivastava, and V. Raghunathan, "Adaptive duty cycling for energy harvesting systems," Proceedings of International Symposium on Low Power Electron. Des., pp. 180-185, Oct 2006.

[17] Y. Jing and H. Jafarkhani, "Single and multiple relay selection schemes and their achievable diversity orders," IEEE Trans. Wireless Commun., vol. 8, pp. 1414 - 1423, Mar. 2009.

[18] M. S. Bahbahani and E. Alsusa, "Relay selection for energy harvesting relay networks using a repeated game," in IEEE Wireless Commun. Netw. Conf. (WCNC), April 2016, pp. 1-6.

[19] N. Newalkar, S. Silakari, and P. K. Shukla, "Comparative analysis of cooperative MAC schemes," in Cloud and Services Computing (ISCOS), 2012 International Symposium on, Dec 2012, pp. 42-48.

[20] P. Liu, Z. Tao, S. Narayanan, T. Korakis, and S. S. Panwar, "CoopMAC: A cooperative MAC for wireless LANs," IEEE J. Sel. Areas Commun., vol. 25, no. 2, pp. 340-354, February 2007.

[21] J. Liu, W. Wang, Z. Zheng, X. Zhang, C. Chen, and X. Shen, "Lifetime extended cooperative MAC protocol for wireless LANs," in Global commun. conf. (GLOBECOM), 2012 IEEE, Dec 2012, pp. 5476-5481.

[22] M. Khalid, Y. Wang, I. h. Ra, and R. Sankar, "Hybrid cooperative MAC protocol for wireless ad hoc networks," in International Conf. Comput. Netw. Commun. (ICNC), Jan 2012, pp. 1015-1019.

[23] F. Liu, T. Korakis, Z. Tao, and S. Panwar, "A MAC-PHY cross-layer protocol for ad hoc wireless networks," in IEEE Wireless Commun. Netw. Conf. (WCNC), March 2008, pp. 1792-1797.

[24] N. Hu, Y. D. Yao, and Z. Yang, "Analysis of cooperative TDMA in rayleigh fading channels," IEEE Trans. Veh. Technol., vol. 62, no. 3, pp. 1158-1168, March 2013.

[25] H. Jiao and Y. L. Frank, "A mini-slot-based cooperative MAC protocol for wireless mesh networks," in 2010 IEEE Globecom Workshops, Dec 2010, pp. 89-93.

[26] A. and H. Y. Kong, "Energy efficient cooperative LEACH protocol for wireless sensor networks," J. Commun. Netw., vol. 12, no. 4, pp. 358365, Aug 2010.

[27] X. Li, M. Chen, and W. Liu, "Application of STBC-encoded cooperative transmissions in wireless sensor networks," IEEE Signal Process. Lett., vol. 12, no. 2, pp. 134-137, Feb 2005.

[28] G. X. Zhang, Pengfei and H.-P. Tan, "Clustering algorithms for maximizing the lifetime of wireless sensor networks with energy-harvesting sensors," Computer Networks, vol. 57, no. 4, pp. 2689-2704, 2013.

[29] M. S. Bahbahani and E. Alsusa, "DC-LEACH: A duty-cycle based clustering protocol for energy harvesting WSNs." IEEE Int. Commun. Conf. (ICC), Submitted, Oct. 2016.

[30] D. Benedetti, C. Petrioli, and D. Spenza, "GreenCastalia: An energyharvesting-enabled framework for the Castalia simulator," Proceedings of ACM ENSSys 2013, pp. 7:1-7:6, Nov 2013. [Online]. Available: http://dl.acm.org/citation.cfm?id=2534215

[31] A. Boulis, "Castalia: A simulator for wireless sensor networks and body area networks user's manual.” NICTA, 2011. [Online]. Available: https://castalia.forge.nicta.com.au

[32] A. Varga and R. Hornig, "An overview of the OMNeT++ simulation environment," in Proceedings of the 1st International Conference on Simulation Tools and Techniques for Communications, Networks and Systems \& Workshops, ser. Simutools '08. ICST, Brussels, Belgium, Belgium: ICST (Institute for Computer Sciences, Social-Informatics and Telecommunications Engineering), 2008, pp. 60:1-60:10. [Online] Available: http://dl.acm.org/citation.cfm?id=1416222.1416290

[33] A. Kansal, J. Hsu, S. Zahedi, and M. B. Srivastava, "Power management in energy harvesting sensor networks," ACM Trans. Embed. Comput. Syst., vol. 6, no. 4, Sep. 2007. [Online]. Available: http://doi.acm.org/10.1145/1274858.1274870

[34] A. Bletsas, A. Khisti, D. Reed, and A. Lippman, "A simple cooperative diversity method based on network path selection," IEEE J. Sel. Areas Commun., vol. 24, no. 3, pp. 659-672, Mar. 2006

[35] S. S. Ikki and M. H. Ahmed, "Performance analysis of cooperative diversity with incremental-best-relay technique over rayleigh fading channels," IEEE Trans. Commun., vol. 59, no. 8, pp. 2152-2161, August 2011.

[36] W. Heizelman, "Application-specific protocol architectures for wireless networks." PhD Thesis, MIT, Jun 2000.

[37] K. Doddapaneni, P. Shah, E. Ever, A. Tasiran, F. A. Omondi, L. Mostarda, and O. Gemikonakli, "Packet arrival analysis in wireless sensor networks," in 2015 IEEE 29th International Conf. on Advanced Information Netw. and Applications Workshops, Mar 2015, pp. 164-169. 